\title{
ANEMIA HEMOLÍTICA CAUSADA POR Ditaxis desertorum (Euphorbiaceae) EM BOVINOS ${ }^{1}$
}

\author{
Carlos Hubinger Tokarnia ${ }^{2}$, Bionô Roque das Chagas ${ }^{3}$, Adenisar Delgado das Chagas ${ }^{4}$ \\ e Heloisa Kreling da Silva ${ }^{5}$
}

\begin{abstract}
Tokarnia C.H., Chagas B.R., Chagas A.D. \& Silva H.K. 1997. [Hemolytic anemia caused by Ditaxis desertorum (Euphorbiaceae) in cattle.] Anemia hemolítica causada por Ditaxis desertorum (Euphorbiaceae) em bovinos. Pesquisa Veterinária Brasileira 17(3/4):112-116. Projeto Saúde Animal-Embrapa/UFRRJ, Km 47, Seropédica, RJ 23851-970, Brazil.

Ditaxis desertorum Pax et K. Hoffm., a herbaceous plant of the Euphorbiaceae family, caused hemoglobinuria due to its hemolytic properties, when force-fed fresh to bovines in daily doses of 1.0 to $2.5 \mathrm{~g} / \mathrm{kg}$; the hemoglobinuria appeared from the 4th to 8 th day of the experiment. After a period of 3 to 5 days of severe hemoglobinuria and anemia, these symptoms disappeared in three of the four animals, although they continued to receive the plant. There was a rapid return to normal blood composition after cessation of the hemoglobinuria. The fourth bovine, which received $2.5 \mathrm{~g} / \mathrm{kg} / \mathrm{day}$, over 5 days, had severe hemoglobinuria and anemia during the last 4 days of life and died on the 8th day. Post-mortem and histopathological examinations revealed hemoglobinuric nephrosis and liver distrophy with centrolobular necrosis of the parenchyma. A single dose of $7.7 \mathrm{~g} / \mathrm{kg}$ or doses of 2.5 and $3 \mathrm{~g} / \mathrm{kg} / \mathrm{day}$ given during 2 days, caused colics and death within a few hours in the three other bovines; post-mortem examinations revealed congestion and oedema of the wall of rumen and reticulum. Case histories indicate that under natural conditions of ingestion only the hemolytic anemia occurs. Although palatable, the ingestion of $\mathrm{D}$. desertorum in sufficient amounts to cause colics and lesions of the forestomachs is probably limited by its caustic properties.
\end{abstract}

INDEX TERMS: Poisonous plants, plant poisoning, Ditaxis desertorum, Euphorbiaceae, cattle, pathology, hemolytic anemia.

SINOPSE.- Ditaxis desertorum Pax et K. Hoffm., planta herbácea da família Euphorbiaceae, causou experimentalmente em bovinos um quadro caracterizado por hemoglobinúria em virtude de sua ação hemolítica, quando administrada por via oral em doses diárias de 1,0 a $2,5 \mathrm{~g} / \mathrm{kg}$ (planta fresca), a partir do $4^{\circ}$ ao $8^{\circ}$ dia do experimento. Após um período de 3 a 5 dias em que os animais tiveram hemoglobinúria e anemia acentuadas, apesar de continuarem a receber a planta (du-

\footnotetext{
${ }^{1}$ Aceito para publicação em 27 de junho de 1997.

2 Depto Nutrição Animal e Pastagem, Universidade Federal Rural do Rio de Janeiro (UFRRJ), Km 47, Seropédica, RJ 23851-970; bolsista do CNPq (305010/ 76-VT).

${ }^{3}$ Médico-Veterinário, Casa Mutirão, Wanderley, Bahia 47940-000.

${ }^{4}$ Médico-Veterinário da Empresa Baiana de Desenvolvimento Agrícola, Wanderley, BA.

${ }^{5}$ Depto Medicina e Cirurgia, UFRRJ.
}

rante um total de 12 a 14 dias), em três dos quatro animais esses sintomas desapareceram. Verificou-se nesses casos uma rápida recuperação dos valores hemáticos logo que cessou a hemoglobinúria. O quarto bovino, que recebeu $2,5 \mathrm{~g} / \mathrm{kg} / \mathrm{dia}$ durante 5 dias, morreu no $8^{\circ}$ dia, tendo apresentado durante os últimos 4 dias de vida hemoglobinúria e anemia acentuadas. À necropsia e nos exames histopatológicos deste animal foram verificadas nefrose hemoglobinúrica e distrofia hepática com necrose centrolobular do parênquima. Dose de 7,7 $\mathrm{g} / \mathrm{kg}$ única ou quantidades de 2,5 e $3 \mathrm{~g} / \mathrm{kg} / \mathrm{dia}$ administradas durante 2 dias seguidos, causaram em três outros bovinos quadro clínico de cólica, com morte em questão de horas, verificando-se à necropsia acentuado edema da parede do rúmen e do retículo. Pelos históricos obtidos somente ocorre, sob condições naturais, a intoxicação caracterizada pelo quadro da anemia hemolítica, indicando que possivelmente a ingestão de $D$. desertorum em quantidades necessárias para 
causar o quadro com lesões dos proventrículos ser, apesar de sua boa palatabilidade, autolimitada pelo efeito cáustico da planta.

TERMOS DE INDEXAÇÃO: Plantas tóxicas, intoxicação por planta, Ditaxis desertorum, Euphorbiaceae, bovinos, patologia, anemia hemolítica.

\section{INTRODUÇÃO}

Em dezembro de 1995, por ocasião de estudos sobre doenças de etiologia obscura possivelmente causadas por plantas tóxicas, na região Oeste do Estado da Bahia, obtivemos no município de Wanderley, na Fazenda Santa Rita, histórico de doença de evolução subaguda em bovinos, em que a urina ficaria com "cor de sangue", e observada de uns 10 anos para cá. Esta doença só ocorreria em determinadas roças onde cresce uma planta herbácea muito pastada pelo gado e da qual o proprietário suspeitava. Trata-se de planta invasora. $\mathrm{O}$ gado se intoxica nas palhadas após a colheita do milho, de maio em diante. A planta continua verde na época da seca. Com 8 dias de permanência nestas roças, já apareceriam os primeiros sintomas da doença. Tirando os animais destas roças no começo da doença, estes se recuperariam logo.

Encontramos grande quantidade da planta apontada pelo proprietário, pastada, e que foi identificada pela Dra. Graziela Maciel Barroso, como Ditaxis desertorum Pax et K. Hoffm., planta da família Euphorbiaceae (Fig. 1 e 2).

Fomos informados que pelo cultivo sucessivo de milho, devido a aração e gradeação, em poucos anos a planta vai desaparecendo. A planta ocorre também na mata, mas em pequena quantidade.

Não encontramos na literatura dados sobre a toxidez de D. desertorum. Consequentemente a planta foi submetida a experimentação em bovinos para se verificar a sua toxidez, determinar as doses tóxicas e o quadro clínico-patológico da intoxicação.

\section{MATERIAL E MÉTODOS}

A planta fresca foi administrada por via oral em diversas dosagens $\mathrm{e}$ durante períodos variáveis, a nove bovinos jovens desmamados. Foram usadas as partes aéreas de Ditaxis desertorum Pax et K. Hoffm. (folhas e galhos finos), que foram coletadas no município de Wanderley, Bahia, em diversas ocasiões, e sempre guardadas em geladeira até o dia de sua administração, no máximo até 6 a 9 dias após a coleta.

Os bovinos de experimentação eram examinados antes do início das administrações da planta e duas vezes ao dia após a primeira administração, com tomada de temperatura, auscultação do coração, pulmão e rúmen, ocasião em que sempre se fazia coleta de amostra de urina, para verificação de hemoglobinúria. No decorrer do experimento era coletado sangue para hematimetria, consistindo na determinação do hematócrito, da hemoglobina e na contagem das hemácias, em alguns animais diariamente, em outros ocasionalmente.

Nos experimentos realizados em Wanderley, os animais durante o dia, eram soltos em um pasto onde eram observados durante parte do dia. Durante a noite eram mantidos em um curral. Nos experimentos realizados em nosso Instituto ( $\mathrm{Km} \mathrm{47,} \mathrm{Rio} \mathrm{de} \mathrm{Janeiro),}$ os animais eram mantidos em baias individuais, com água e capim picado de capineira à vontade. Na parte da manhã eram soltos em um pequeno piquete.

Finalmente foram realizados experimentos com a planta dessecada, também em bovinos jovens, em nosso Instituto.

\section{RESULTADOS}

Ditaxis desertorum revelou-se tóxica para bovinos em nossos experimentos. Dependendo das doses administradas, causou sintomatologias distintas de intoxicação (Quadro 1). Dose elevada $(7,7 \mathrm{~g} / \mathrm{kg})$ única ou doses médias $(2,5$ e $3 \mathrm{~g} / \mathrm{kg})$ repetidas em 2 dias seguidos, causaram um quadro clínico de cólica com morte em questão de horas. Os achados de necropsia consistiram, principalmente, em alterações do aparelho digestivo. Havia acentuado edema da parede do rúmen, sobretudo nas regiões próximas ao sulco esofagiano, e da parede do retículo. As papilas do rúmen estavam avermelhadas e em algumas áreas se podia retirar fragmentos da camada epitelial com facilidade, deixando exposta a própria também avermelhada. A mucosa do abomaso em dois dos três animais (Bov. 5211 e 5505) esteve levemente avermelhada. Havia ainda leve esplenomegalia e leve ascite. Os exames histopatológicos revelaram principalmente lesões na parede do rúmen sob a forma de desprendimento do epitélio, congestão da própria, edema da submucosa acompanhado de congestão e hemorragias, edema na muscular e da serosa. Em um animal (Bov. 5503) o edema na muscular e serosa era acompanhado de congestão e hemorragias, e em dois animais (Bov. 5503 e 5505) havia infiltrados polimorfonucleares que no Bovino 5503 eram acentuados, nas áreas com edema.

Havia ainda acentuada congestão do baço nos três animais (Bov. 5211, 5503 e 5505) e em um animal (Bov. 5505) leve congestão das partes profundas da própria e na submucosa do abomaso.

Doses menores, de $1,0 \mathrm{~g} / \mathrm{kg}, 1,5 \mathrm{~g} / \mathrm{kg}$ e $2,0 \mathrm{~g} / \mathrm{kg}$, e em um bovino, também a de $2,5 \mathrm{~g} / \mathrm{kg}$, administradas diariamente, causaram um quadro caracterizado por hemoglobinúria e anemia a partir do $4^{\circ}$ ao $8^{\circ}$ dia do experimento. $O$ animal (Bov. 5225), que ingeriu $2,5 \mathrm{~g} / \mathrm{kg} / \mathrm{dia}$, durante 5 dias (a administração da planta foi interrompida quando o animal mostrou anorexia), morreu no $8^{\circ}$ dia do experimento. Este animal apresentou a partir de $1 \mathrm{~h} 54 \mathrm{~min}$ após o fim da primeira administração da planta, durante aproximadamente 8 horas, sinais de cólica, que não mais foram observados após as administrações seguintes. Teve hemoglobinúria e anemia acentuadas a partir do $4^{\circ}$ dia do experimento até o dia da morte, no $8^{\circ}$ dia (Fig. 3). Nos três outros animais (Bov. 5501, 5502 e 5508) após um período de 3 a 5 dias em que tiveram hemoglobinúria e anemia acentuadas, apesar de continuarem a receber a planta, num período total de 12 a 14 dias, esses sintomas desapareceram. Verificou-se uma rápida recuperação dos valores hemáticos logo que cessou a hemoglobinúria. Em nenhum animal foi verificado icterícia. O soro/plasma sanguíneo se apresentava hemolisado durante a fase da hemoglobinúria.

Porém o bovino que recebeu $2,5 \mathrm{~g} / \mathrm{kg} /$ dia durante 12 dias (Bov. 5502), ao contrário dos outros dois animais, após a cessa- 
Quadro 1. Intoxicação experimental pelas partes aéreas frescas de Ditaxis desertorum em bovinos (coletadas em 2.12.95, 28.2 e 6.3.97, na Faz. Santa Rita, mun. Wanderley, Bahia) Bovino Peso Primeiro Doses Desfecho Ińcio Evolução Iń́cio Evolução Morte Hemoglobinúrial _ Dias do experimento

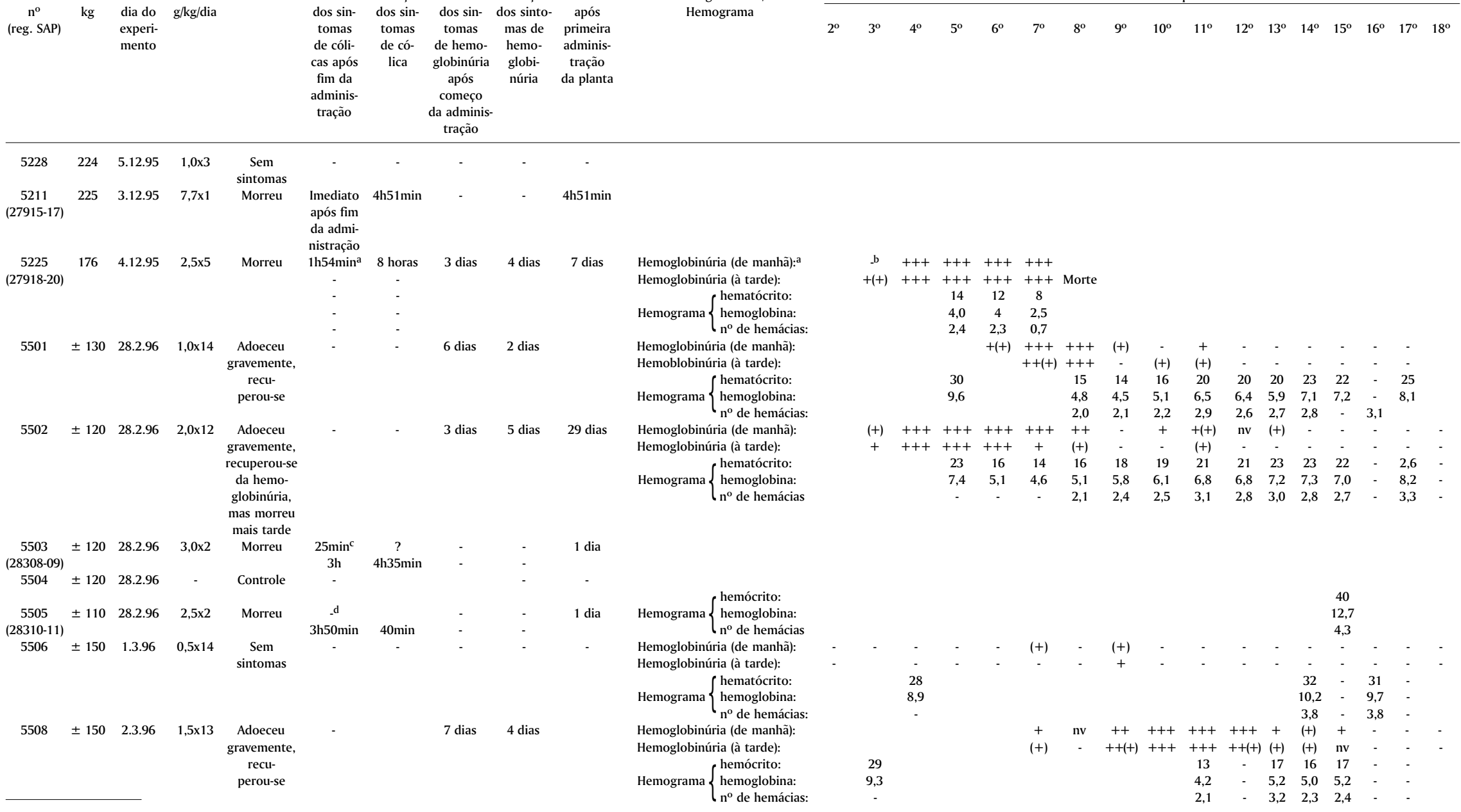

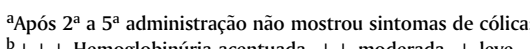

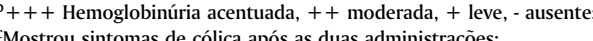
dMostrou sintomas de cólica somente após a $2^{2}$ admininistração. 


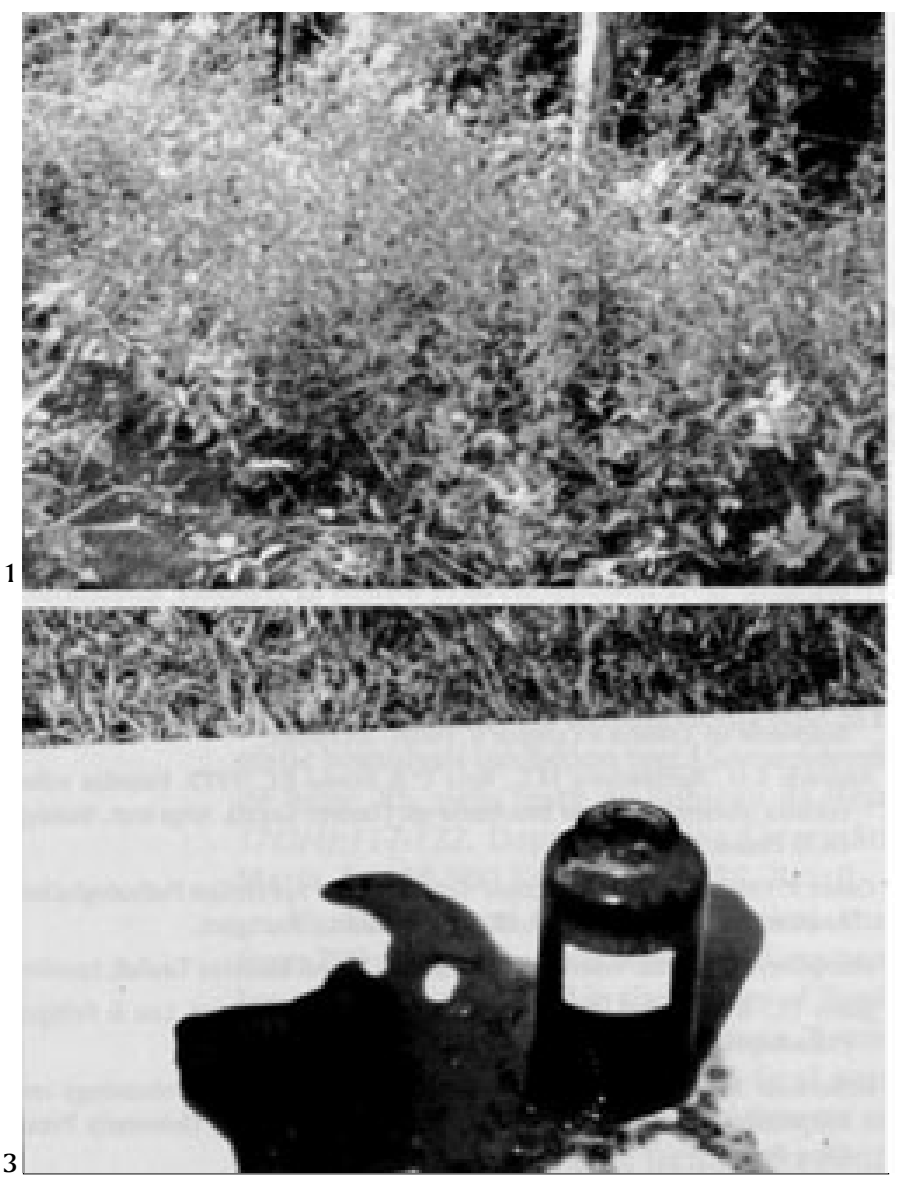

ção da hemoglobinúria e recuperando-se da anemia, começou a mostrar diminuição do apetite, emagrecimento e pêlos arrepiados, e no $30^{\circ}$ dia do experimento foi encontrado morto. Os outros dois animais (Bov. 5501 e 5508) nunca perderam o apetite.

Os achados de necropsia no único animal (Bov. 5225) que morreu durante a fase de hemoglobinúria, foram mucosas pálidas (esbranquiçadas), sangue aquoso, bexiga contendo urina com cor de vinho tinto, rins aumentados de volume com consistência de borracha, córtex renal na superfície e ao corte com coloração marrom-escura, esplenomegalia moderada, fígado na superfície e ao corte de cor alaranjada e com aspecto mosqueado, vesícula biliar estendida por bile verdegrossa e mucosa, conteúdo do rúmen, omaso, ceco e cólon ressequido.

As principais alterações histológicas foram observadas no fígado e rim. No fígado havia necrose de coagulação centrolobular acentuada, as células hepáticas na periferia da área de necrose apresentando processo de degeneração hidrópico-vacuolar (Sudan III negativo); os hepatócitos ao redor do espaço porta apresentavam pequenos vacúolos (Sudan III positivo). No rim foi observada grande quantidade de cilindros densos, de coloração vermelha levemente marrom, na luz dos túbulos uriníferos, e esferas semelhantes nos espaços de Bowman; as células epiteliais de muitos túbulos uriníferos apresentavam numerosas gotas de diversos tama-

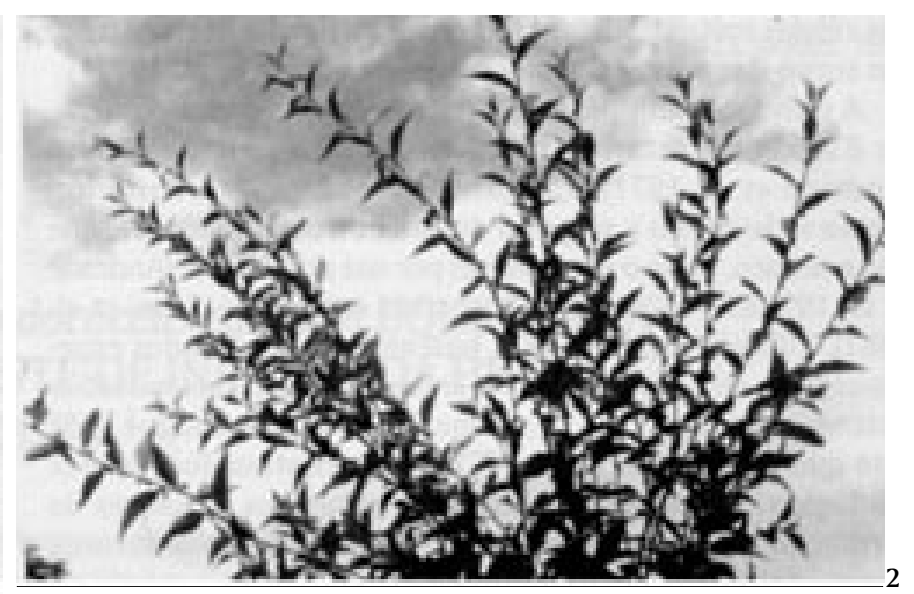

Fig. 1. Ditaxis desertorum, no município de Wanderley, Bahia.

Fig. 2. D. desertorum em floração.

Fig. 3. Hemoglobinúria acentuada, causada por $D$. desertorum, confere à urina dos bovinos coloração comparável a de Coca-Cola.

nhos de substância da mesma natureza. Ainda havia nos espaços de Bowman e na luz de túbulos uriníferos substância albuminosa sob forma de esferas e cilindros. No baço foi constatada congestão acentuada.

Pela dessecagem a planta perdeu toda sua toxidez. (Quadro 2)

\section{DISCUSSÃO}

As causas da anemia hemolítica, que é o resultado da destruição excessiva de hemácias circulantes, podem ser infecciosas, tóxicas ou imunológicas. Entre as tóxicas estão as causadas por plantas. Destas, são bastante conhecidas no hemisfério norte as intoxicações por Brassica spp., Allium spp. e Mercurialis spp. (Stöber 1970, Jones \& Hunt 1983, Humphreys 1988, Kellerman et al. 1988, Radostits et al. 1994).

No Brasil, até agora somente conhecíamos Brachiaria radicans como pertencente a este grupo de plantas. Esta intoxicação se caracteriza por uma coloração marromavermelhada da urina, andar desequilibrado, mucosas pálidas e micções frequentes. Há anemia hemolítica e metemoglobinemia, elevado teor de nitrito no soro sanguíneo e hemoglobinúria. Os achados de necropsia consistem em rins tumefeitos e de coloração marrom. Histologicamente foram descritas, no rim, presença de hemoglobina nos espaços de Bowman, na luz dos túbulos uriníferos e no citoplasma 
das células epiteliais desses túbulos, e no fígado, micronecrose em torno das veias centrolobulares. (Andrade et al. 1971a)

A princípio admitiu-se a hipótese de que o efeito nocivo de $B$. radicans estaria ligado ao seu alto teor de nitrato (Andrade et al. 1971a,b,c, Rosenfeld et al. 1971). Posteriormente se levantou a suspeita de que outro componente, que não o nitrato, fosse o responsável por sua ação tóxica (Andrade et al. 1972a,b, 1973, 1975, Rosenfeld et al. 1976, Lessi et al. 1981). Villalobos et al. (1981), baseando-se em estudos experimentais, concluíram que a sintomatologia, os achados anatomo-patológicos e os exames químicos do sangue indicam que na intoxicação por $B$. radicans existem dois fatores etiológicos importantes: a) os nitratos-nitritos indutores da formação de metemoglobina e b) um ou mais fatores deconhecidos responsáveis pelo dano hepato-renal e a hemólise intravascular.

Um aspecto interessante da intoxicação por Ditaxis desertorum em nossos experimentos foi que a mesma planta produziu dois quadros clínico-patológicos diferentes, na dependência da dosagem da planta ingerida; um quadro caracterizado por hemoglobinúria, em virtude de sua ação hemolítica, e outro, de cólica, devido o seu efeito cáustico.

As lesões renais encontradas no único bovino que morreu em função da ação hemolítica da planta, são descritas na literatura como nefrose hemoglobinúrica (Trautwein 1991). Em relação às lesões do fígado, Cohrs (1962) menciona como um fator importante das distrofias hepáticas, a hipoxidose; a necrose intralobular, principalmente distrofia hepática centrolobular com necrose, ocorre, de acordo com este autor, especialmente naquelas doenças em que há rápida e forte hemólise. Também Rubarth (1967) menciona anemia, e entre elas a anemia hemolítica, como causa de alterações hepáticas centrolobulares.

O outro aspecto importante foi que os animais que apresentaram o quadro clínico-patológico da anemia hemolítica se recuperaram após poucos dias (com uma exceção), apesar de continuarem a ingerir a planta. Pode-se pensar no desenvolvimento de um mecanismo no animal, no rúmen ou no fígado, pelo qual o princípio tóxico responsável pela anemia hemolítica fosse desativado.

Pelos históricos obtidos parece que sob condições naturais somente ocorre a intoxicação caracterizada pelo quadro de anemia hemolítica. Apesar de a planta aparentemente ter boa palatabilidade, pode se especular que devido ao seu efeito cáustico, demonstrado pelas lesões no rúmen e retículo quando administrada em quantidades maiores, os animais sob condições naturais não comem grandes quantidades da planta $(2,5 \mathrm{~g} / \mathrm{kg}$ ou mais) por dia.

Agradecimentos.- À Dra. Graziela Maciel Barroso, Jardim Botânico do Rio de Janeiro, pela identificação da planta; ao Assistente de Pesquisa João Luiz Bastos pela valiosa colaboração nos trabalhos de experimentação com a planta em Wanderley/Bahia; e aos criadores de Wanderley, que puseram os bovinos e as instalações a nossa disposição e forneceram históricos.

\section{REFERÊNCIAS}

Andrade S.O., Peregrino C.J.B. \& Aguiar A.A. 1971a. Estudos sobre Brachiaria sp. (Tanner Grass). I. Efeito nocivo para bovinos. Arqs Inst. Biológico, S. Paulo, 38(3):135-150.

Andrade S.O., Retz L. \& Marmo O. 1971b. Estudos sobre Brachiaria sp. III. Ocorrências de intoxicações de bovinos durante um ano (1970-1971) e níveis de nitrato em amostras de gramínea. Arqs Inst. Biológico, S. Paulo, 38(4):239-252.

Andrade S.O., Retz L. \& Velloso C.A. 1971c. Estudos sobre Brachiaria sp. (Tanner Grass). II. Dosagens de nitrato em soro de bovinos. Arqs Inst. Biológico, S. Paulo, 38(3):151-161.

Andrade S.O., Cosentino J.R. \& Scott W.N. 1972a. Estudos sobre novilhas alimentadas diariamente com milho ou melaço e Brachiaria sp. (Tanner Grass). Arqs Inst. Biológico, S. Paulo, 39(4):329-333.

Andrade S.O., Figueiredo A.L. \& Nothenberg M.S. 1972b. Estudos sobre Brachiaria sp. IV. Investigações sobre o efeito da gramínea, apresentando teor baixo de nitrato, em ovinos, bovinos e equinos. Arqs Inst. Biológico, S. Paulo, 39(4):257-261.

Andrade S.O., Retz L. \& Nothenberg M.S. 1973. Studies on the toxic effect of Brachiaria sp. Ciência e Cultura, S. Paulo, 25(7):643.

Andrade S.O., Nothenberg M.S., Retz L. \& Bueno P.C. 1975. Estudos sobre cobaias alimentadas com Brachiaria sp. (Tanner Grass). Arqs Inst. Biológico, S. Paulo, 42:243-246.

Cohrs P. 1962. Nieberle und Cohrs' Lehrbuch der Speziellen Pathologischen Anatomie der Haustiere. 4.Aufl. Gustav Fischer, Stuttgart.

Humphreys D.J. 1988. Veterinary Toxicology. 3rd ed. Baillière Tindall, London. Jones T.C. \& Hunt R.D. 1983. Veterinary Pathology. 5th ed. Lea \& Febiger, Philadelphia.

Kellerman T.S., Coetzer J.A.W. \& Naudé T.W. 1988. Plant Poisonings and Mycotoxicoses of Livestock in Southern Africa. Oxford University Press, Cape Town.

Lessi E., Vianna J.R. \& Rodrigues P.C. 1981. Contribuição ao estudo da toxidez ocasional da Brachiaria sp. (Tanner Grass) em bovinos. Revta Bras. Med. Vet. 4(4):32-37.

Radostits O.M., Blood D.C. \& Gay C.C. 1994. Veterinary Medicine. Baillière Tindall, London.

Rosenfeld G., Reichmann C.E. \& Andrade S.O. 1971. Anemia hemolítica em bovinos alimentados com Brachiaria sp. (Tanner Grass). Arqs Inst. Biológico, S. Paulo, 38(4):267-273.

Rosenfeld G., Reichmann C.E., Faria L.J. \& Andrade S.O. 1976. Estudos sobre o quadro hemático de ovinos e equinos em pastagem de Brachiaria radicans Napper (Tanner Grass) no período do inverno. Arqs Inst. Biológico, S. Paulo, 43(3/4):99-103.

Rubarth S. 1967. Leber und Gallenwege, p. 1-299. Hypoxämische Leberveränderungen, p. 103-104. In: Dobberstein J., Pallaske G. \& Stünzi H. (ed.) Handbuch der Speziellen Pathologischen Anatomie der Haustiere, begründet von E. Joest. Band VI. Digestionsapparat, II. Teil. 3. Aufl. Paul Parey, Berlin.

Stöber M. 1970. Pflanzenbedingte Schädigungen der Kreislauforgane, des Blutes oder der Blutbildung, p. 1255-1265. In: Rosenberger G. (ed.) Krankheiten des Rindes. Paul Parey, Berlin.

Trautwein G. 1991. Harnorgane, p. 517-575. In: Schulz L.-C. (ed.) Pathologie der Haustiere. Teil I. Organveränderungen. Gustav Fischer, Jena.

Villalobos S.J., Meneses G.A., León C.S. \& Carballo C.G. 1981. Clinica y patologia de la intoxicacion con Brachiaria radicans Napper (Tannergrass). Cienc. Vet., Costa Rica, 3:163-169. 